\title{
Real Time Vehicle Recognition: A Novel Method for Road Detection
}

\author{
Adrián Peñate Sánchez², Alexis Quesada Arencibia ${ }^{1}$, Carlos M. Travieso González \\ ${ }^{1}$ Institut de Robòtica i Informàtica Industrial, (CSIC-UPC), Barcelona, Spain \\ ${ }^{2}$ Institute for Cybernetics, University of Las Palmas de Gran Canaria, Las Palmas de G.C., \\ Las Palmas, España
}

\begin{abstract}
Knowing the location of the road in an intelligent traffic systems is one of the most used solutions to ease vehicle detection. For this purpose we propose a vehicle recognition algorithm which performs a real time automatic detection of the zones which vehicles occupy. Such algorithm is capable of functioning under extreme conditions such as low resolution, low capture angle and gray scale images.
\end{abstract}

Keywords: Automatic road detection, Intelligent transport systems, real time vehicle recognition.

\section{Introduction}

The purpose of this work has been the real time segmentation of vehicles in traffic environments using low resolution videos in gray scale. The method used to resolve this problem has been background subtraction with an adaptive background algorithm to learn what the background is in each moment, with this we intend to solve the problems produced by changes in the illumination.

We have developed a new method to minimize the erroneous segmentation of objects; such method consists in the determination of the probability associated with each pixel in order for it to contain a vehicle when it is segmented. These probabilities are used to relax the threshold in zones where the probability of finding a vehicle is higher. If we place the adequate global threshold we will avoid numerous false positive detections in the zones where vehicles do not transit.

The method we propose can be easily used to perform tracking by detection which has proven to be a very robust approach beacause it does not suffer from intra-frame errors due to the propagation of the error.

We are going to work with the videos used by Neeraj K. Kanhere and Stanley T. Birchfield in their paper [1], and that where kindly lent to us by them. These videos 
are in a $320 \times 240$ resolution with a low angle and in gray scale. We have also made use of [2] for our Matlab development.

\section{Image Segmentation}

We have used background subtraction for each frame of the videos. To determine the initial background we settle it as the average of the first 200 frames. From this point we use the adaptive algorithm described in [3] that uses only non segmentated pixels in the background updating process.

Once extracted the background we use a similar methodology as the one described in [4], the difference consists in that we repeat the process twice, first over the segmented image in gray scale and then, after using the threshold, over the binary image that results. We also differ with [4] in that the second time we use a simple dilation and a simple erosion instead of a double closing and a double opening, and we end with a filling.

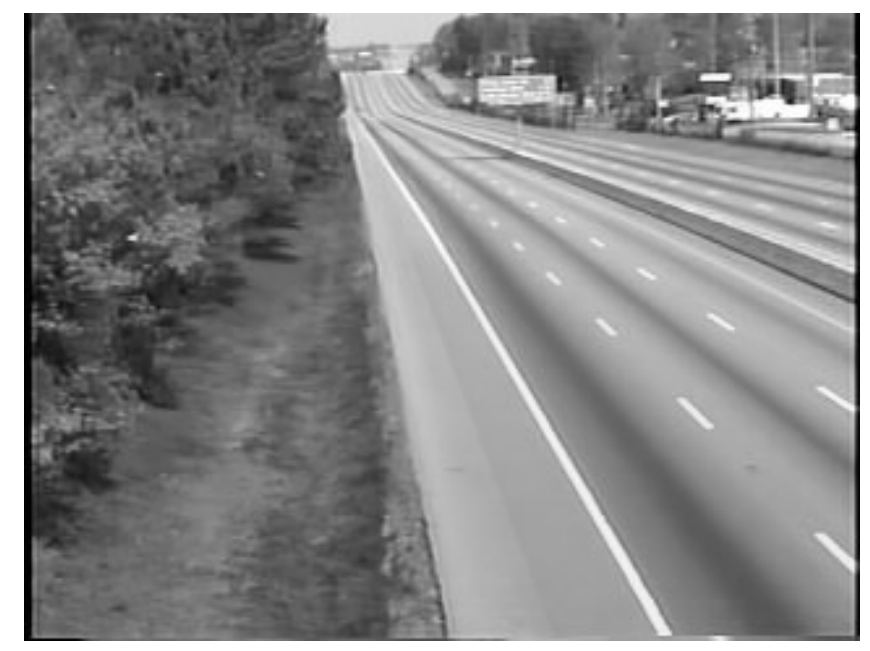

Fig. 1. Background obtained after applying the proposed techniques.

Once we have obtained the blobs in the image we calculate their intrinsic parameters such its are, maximum values of $\mathrm{x}$ and $\mathrm{y}$, the centroid and its orientation. We show over the original image a bounding box that shows the segmentation of each vehicle. 
This process is thought in order to make it ready to use the temporal inference described in [4] to perform tracking and determine the grouping or splitting of blobs in critical cases such as those when partial occlusion happens between to vehicles due to perspective or shadows, or when not all blobs of the same vehicle have been grouped.

\section{Segmentation process:}

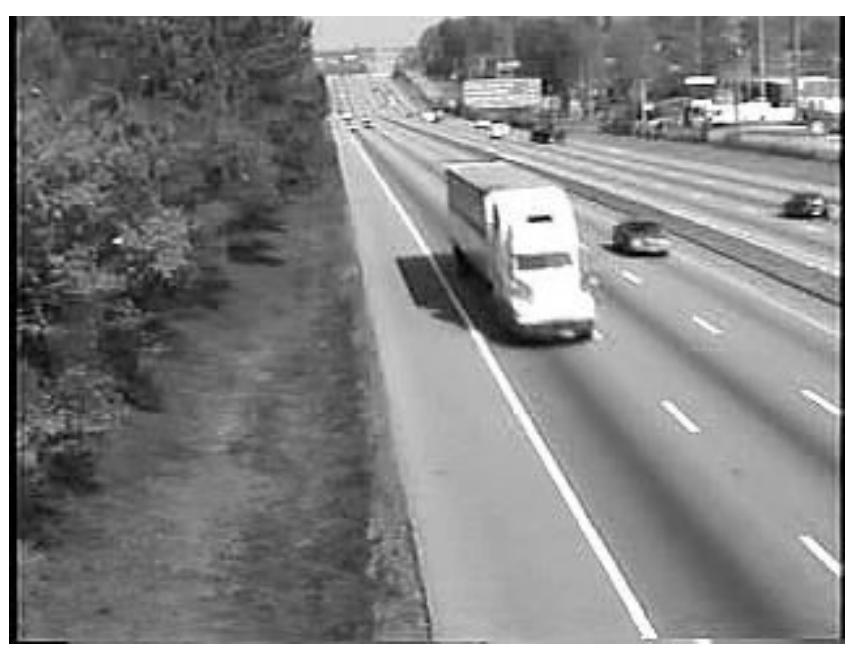

Fig. 2. Original image.

We are going to do a walk-through the whole segmentation process. In Fig. 2. we can see the original image as in one of the frames of the database of [1]. The first step would be to subtract the background as seen in Fig. 1. from the image we are going to process, the result can be seen in Fig. 3. As can be seen the result of having produced a good estimation of the background pays off at this point.

The resting part is to perform the clustering of the image in the two main steps we have mentioned earlier, once this has been done we apply the threshold to the image, we obtain the binary image, we perform the final morphological operations and we retrieve the final bloobs of the image. 


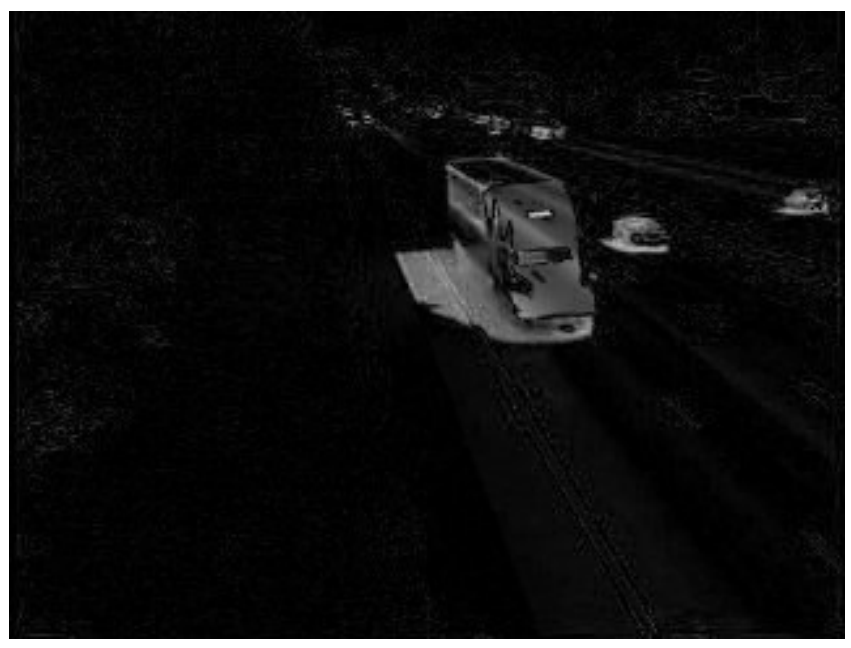

Fig. 3. Step 2, result of subtracting the background from the original image.

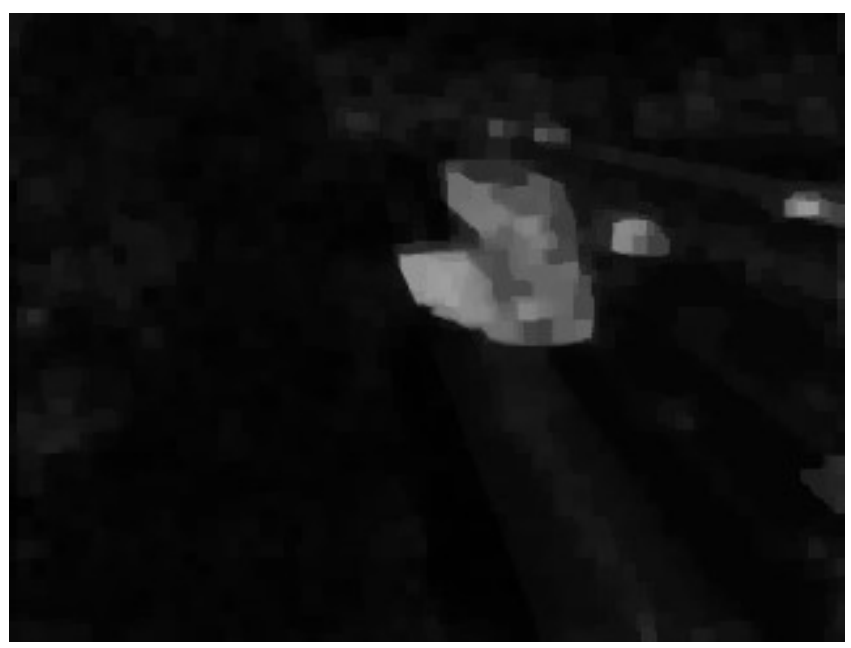

Fig. 4. Step 3, result of applying the morphological operations of filling, closing and opening. 


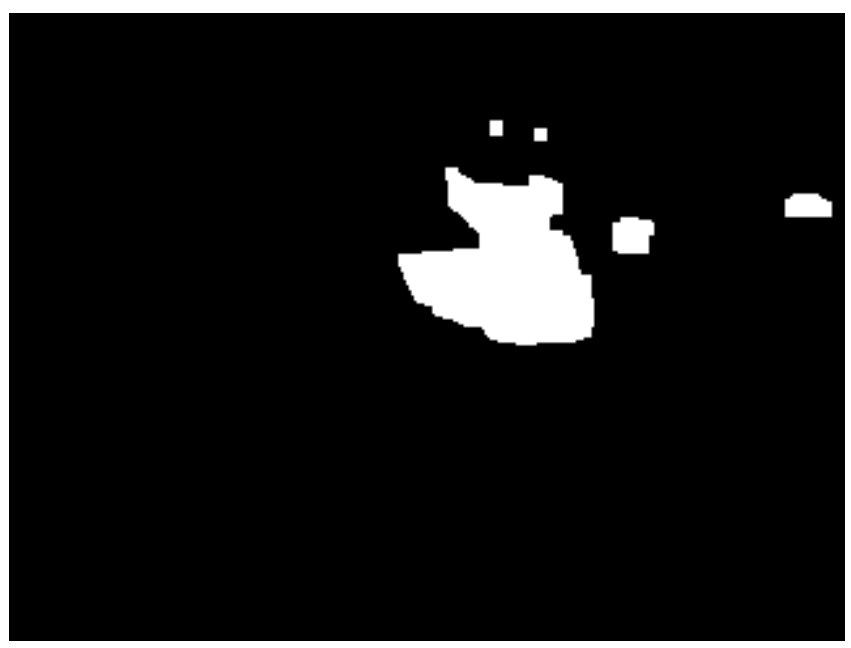

Fig. 5. Step 4, Final phase of threshold and dilation and erosion operations.

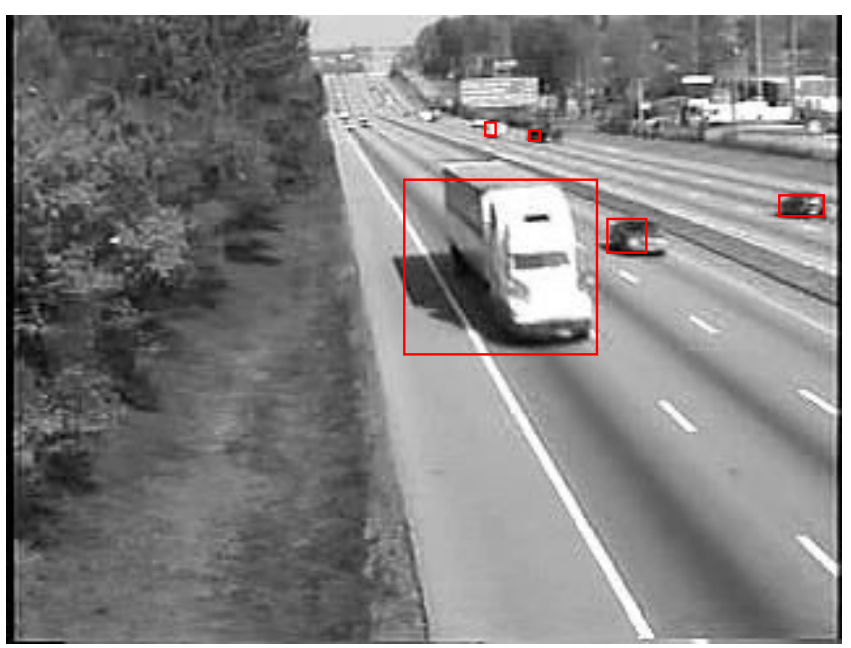

Fig. 6. Final result of the segmentation and tracking by detection. 


\section{Automatic Segmentation Zone Detection}

A recurrent problem in most segmentation algorithms for traffic is appearance of noise caused by moving objects in the background, trees that are moved by the wind, pedestrians crossing a road, and so on. In contexts like this one in which objects of interest occupy a place in the frame that is completely different than the other objects that are moving, we can presuppose that it will be in this areas that we will find our objects of interest.

To find the areas of vehicle transit, which will be the road, we suppose that they will be large areas in which the majority of the movement will be focussed. To enhance this zones we will apply a different threshold to them than to the rest of the environment.

The areas that we will enhance will be those that have a probability value higher than the mean value, and that have a big size, with this we avoid the problem derived from the movement of leaves by the wind. In order for the probability mask to be initialized properly it is necessary not to impose a very restrictive threshold to the zones that are not of interest to us.

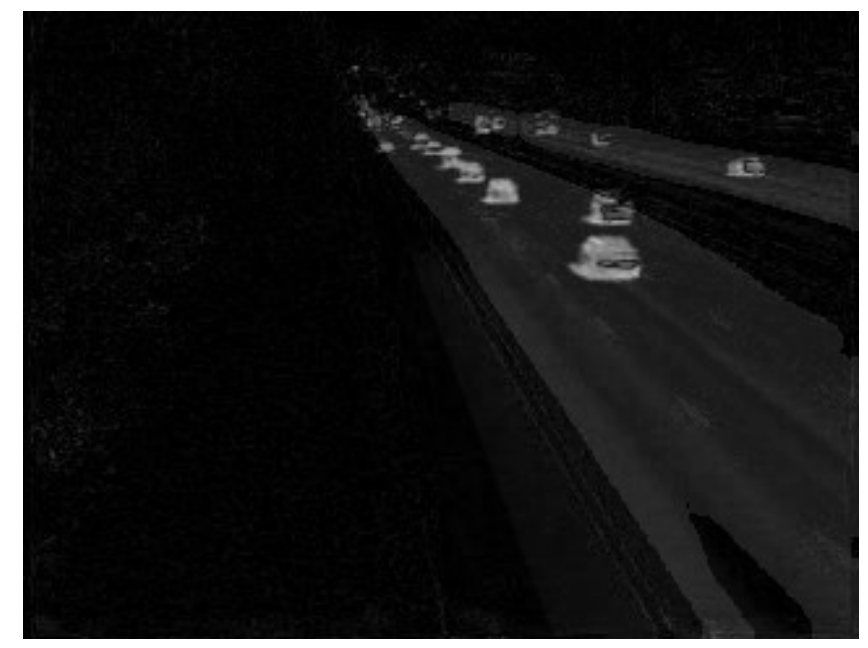

Fig. 7. Result of applying the probabilistic mask over the substracion of the background. 
In Fig. 7. we can observe how by applying the mask we propose the areas that contain more relevant information, pop-up from the rest hence enhancing the detection of vehicles and avoiding the detection of other moving objects such as pedestrians.

The process for the calculation of the probability mask will be to calculate and average with the previous segmented images, applying afterwards the threshold. With the binary image we will apply an erosion followed by a dilation and a filling at the end. In conclusion the novelty of our work consists in applying image processing techniques to probability distributions that enables us to detect the roads in our images in a quite consistent way.

\section{References}

1. Real-Time Incremental Segmentation and Tracking of Vehicles at Low Camera Angles Using Stable Features. Neeraj K. Kanhere and Stanley T. Birchfield, Senior Member, IEEE.

2. Machine Vision Toolbox. Peter I. Corke. peter.corke@csiro.au

3. Neural-edge-based vehicle detection and traffic parameter extraction. D.M. Ha*, J.-M. Lee, Y.-D. Kim.

4. Multilevel Framework to Detect and Handle Vehicle Occlusion. Wei Zhang, Q. M. Jonathan Wu, Member, IEEE, Xiaokang Yang, Senior Member, IEEE, and Xiangzhong Fang. 